\title{
Durable, Washable and Flexible Conductive PET Fabrics Designed by Fibre Interfacial Molecular Engineering
}

DOI:

10.1002/mame.201600234

\section{Document Version}

Accepted author manuscript

Link to publication record in Manchester Research Explorer

\section{Citation for published version (APA):}

Liu, X., Guo, R., Shi, Y., Deng, L., \& Li, Y. (2016). Durable, Washable and Flexible Conductive PET Fabrics

Designed by Fibre Interfacial Molecular Engineering. Macromolecular Materials and Engineering, 301(11), 13831389. https://doi.org/10.1002/mame.201600234

\section{Published in:}

Macromolecular Materials and Engineering

\section{Citing this paper}

Please note that where the full-text provided on Manchester Research Explorer is the Author Accepted Manuscript or Proof version this may differ from the final Published version. If citing, it is advised that you check and use the publisher's definitive version.

\section{General rights}

Copyright and moral rights for the publications made accessible in the Research Explorer are retained by the authors and/or other copyright owners and it is a condition of accessing publications that users recognise and abide by the legal requirements associated with these rights.

\section{Takedown policy}

If you believe that this document breaches copyright please refer to the University of Manchester's Takedown Procedures [http://man.ac.uk/04Y6Bo] or contact uml.scholarlycommunications@manchester.ac.uk providing relevant details, so we can investigate your claim.

\section{OPEN ACCESS}




\section{WILEY-VCH}

DOI: $10.1002 /(($ please add manuscript number $))$

\section{Article type: (Full Paper)}

Durable, Washable and Flexible Conductive PET Fabrics Designed by Fibre Interfacial Molecular Engineering

Xuqing Liu ${ }^{*}$, Ruisheng Guo ${ }^{b}$, Yuxi Shi ${ }^{a}$, Libo Deng ${ }^{c}, Y_{i}$ Li $^{* a}$

Dr.X.Q. Liu, Yuxi Shi, Prof.Y.Li,

School of Materials, University of Manchester, Oxford Rd, Manchester, M13 9PL, UK

E-mail: Xuqing.liu@manchester.ac.uk,henry.yili@manchester.ac.uk

Dr. R.S. Guo

State Key Laboratory of Solid Lubrication, Lanzhou Institute of Chemical Physics, Chinese Academy of Sciences, Lanzhou 730000, China.

Dr. L.B. Deng

College of Chemistry and Environmental Engineering, Shenzhen University, Shenzhen 518060, China

Keywords: (fibre interface, polymer brushes, copper electroless deposition, conductive fibre)

\section{Abstract:}

Conductive fibres and fabrics are an essential part of wearable electronics and devices.

How to fabricate durable conductive substrate is still a huge challenge to the application and commercialization of wearable electronics. Here we report an effective and economic process to obtain conductive yarns and fabrics localized copper plating onto synthesized polymers: poly(ethylene terephthalate) (PET). The active interface was created via plasma treatment to generate active chemical groups on PET substrate, which reacted with ATRP initiator for the following polymerization. The Pd ion entrapped by polymer brushes are then reduced in situ and the $\mathrm{Pd}^{0}$ species act as a catalytic seed layer for following electroless copper nanoparticles growth on the active interface. Scanning electron microscopy (SEM), X-ray diffraction (XRD), Field Emission Gun Scanning Electron Microscopy (FE-SEM) and Atomic force 


\section{WILEY-VCH}

microscopy (AFM) were used to characterize the samples in this process, and the copper loading was quantified by weight. The morphology and composition analysis show that the copper coating on PET fabrics is compact and continuous, which leads to excellent electronic conductivity. The copper coating obtained in this polymer brushes bridged process can pass through the hand washing challenge, and shows excellent adhesion with PET substrate.

\section{Introduction}

Wearable electronics and devices show widely potential applications such as communications, medical monitoring, costume lights and computing devices, this attracts numerous interest from fashion designers, costumers, and scientists.

Seeking an economical technology to fabricate conductive fibres with high conductivity and long-term durability is an essential and critical element for the commercialization of wearable devices and electronics. Poly(ethylene terephthalate) (PET) is widely used as a commercial flexible substrates because it is cheap and highly flexible with chemical stability. A great interest in the deposition of metallic layers on flexible PET substrate has gradually increased for electrical conductivity such as the chips on flex packaging, smart textiles, health care and electromagnetic shielding effectiveness ${ }^{[1,2-5]}$. As an electrical connector, long-term reliability, high endurance and durability of these composites is inherently related to the adhesion between metals coating and polymer substrates. ${ }^{[6,7]}$

There are various methods to metalize PET surfaces, such as physical vapor deposition, magnetron sputtering, chemical vapor deposition and electroplating plating ${ }^{[3-6,8]}$. But there are no chemical bonds or other tethering force between copper and PET substrate, which fabricated via the above-declared approaches. Nevertheless, the inherently poor adhesion between metal particles and polyester represents a bottleneck in its widespread application under usable conditions, knowledge and mechanism of the interface of $\mathrm{Cu} / \mathrm{PET}$ system remain insufficient $^{[2,3,9]}$. Additionally, for PET substrates with particular 3D structure, such as PET fabrics, uniformity and continuity of the deposited metal films by physical vapor deposition 


\section{WILEY-VCH}

and chemical vapor deposition will be affected by the 3D structure as a spatial mask, which will lead to poorly containable electro-conductivity ${ }^{[5,7,10]}$.

Accordingly, this study is aimed at exploring a high effective approach for preparing electrically conductive yarns/fabrics with high endurance and durability by electroless deposition (ELD) of copper nano-particles onto PET fabrics, which is modified by polyelectrolyte brushes as an adhesion layer. In detail, the first step deals with plasma treatment for PET substrate activation so as to increase reactive chemical groups, such as hydroxyl group, carbonyl group and amino group, thereby facilitating the grafting polyelectrolyte brushes onto the PET substrate surface. The second step is to modify PET substrate by polyelectrolyte brushes via surface-initiated atomic transfer radical polymerization (SI-ATRP) $)^{[11]}$, and the stretched polyelectrolyte brushes in aqueous solution anchored the catalyst. The third step concerns the captured catalyst activating electroless plating metallization.

The composition and topography of prepared copper films were characterized by scanning electron microscopy (SEM), X-ray diffraction (XRD) and atomic force microscopy (AFM), respectively. Modified PET yarns/fabrics show high conductivity and can be used as electrical wires for powering light-emitting diodes (LEDs). Importantly, the polymer brushes bridged metal/PET hierarchical structures provide electrical durability under many washing cycles. In principal, this effective approach can be extended as a general method for fabricating conductive yarns and fabrics from all kinds of synthetic fibres.

\section{Experimental Procedure}

\subsection{Materials}

Poly(ethylene terephthalate) (PET) films were supplied by DuPont Company. Ammonium tetrachloropalladate(II) $\quad\left(\left(\mathrm{NH}_{4}\right)_{2} \mathrm{PdCl}_{4}\right), \quad$ 2-(methacryloyloxy)ethyl-trimethylammonium chloride (METAC) (80 wt.\% aqueous solution) and all other chemicals were purchased from Sigma-Aldrich. PET fabrics were commercial textile purchased from markets. Each PET 


\section{WILEY-VCH}

fabric was ultrasonically cleaned in acetone, and distilled water for 3 times, respectively, then dried with nitrogen gas stream.

\subsection{Surface Plasma Treatment}

To graft PMETAC brushes on the surfaces of PET, initial substrates are firstly activated by oxygen plasma treatment. The treated PET surface, anchoring on its functional groups such as hydroxyl, carbonyl, carboxylic, amino and amines, and the functional groups generated can be confirmed by the change of contact angle testing. The PET surface was modified to provide chemical functionality to graft initiator for $\mathrm{ATRP}^{[12]}$, significantly altering from the hydrophobic to hydrophilic. In this article, the formed chemical groups anchored the initiator and substantial polymer brushes, to greatly enhance adhesive strength and permanency of the conductive coating on the PET fabrics as well.

\subsection{Immobilization of ATRP Initiator}

Cleaned PET substrates were firstly immersed into a solution containing $5 \mathrm{mM} 3-$ (trichlorosilyl) propyl 2-bromo-2-methylpropanoate (ATRP initiator) in anhydrous toluene for 1h, allowing the active groups initiated by plasma to react with the trichlorosilane groups. Then PET substrate was cleaned by fresh anhydrous toluene and acetone several times to remove any physisorbed initiators, heated subsequently drying in a vacuum at $60{ }^{\circ} \mathrm{C}$ consistently for $1 \mathrm{~h}$.

\subsection{Surface-Initiated ATRP}

As classical polymerization, $18.4 \mathrm{~g}$ of 2-(methacryloyloxy)ethyl-trimethylammonium chloride (METAC) was dissolved in $20 \mathrm{~mL}$ of $\mathrm{MeOH}$ and degassed by passing a continuous stream of pure $\mathrm{N}_{2}$ through the solution while being stirred for (20 min). 2,2-Dipyridyl (0.96 g), $\mathrm{Cu}(\mathrm{I}) \mathrm{Br}$ $(0.24 \mathrm{~g})$, and $\mathrm{Cu}(\mathrm{II}) \mathrm{Br}_{2}(0.038 \mathrm{~g})$ were sequentially added to above solution. With a stream of dry $\mathrm{N}_{2}$, the mixture solution was then stirred consistently and degassed for $15 \mathrm{~min}$. Subsequently, initiator modified PET substrates were immerged in Schlenk tubes, degassed (4 $\times$ high-vacuum pump $/ \mathrm{N}_{2}$ refill cycles), and reacted at $30^{\circ} \mathrm{C}$ under $\mathrm{N}_{2}$ atmosphere. The mixed 


\section{WILEY-VCH}

solution was syringed into each Schlenk tube, adding enough solution to submerge each sample completely. The samples remained in the solution for polymerization. After polymerization, the samples were removed, washed with methanol and water, and then dried under a stream of air.

\subsection{Metallization by Electroless Deposition (ELD)}

The modified substrates were placed in the dark for $15 \mathrm{~min}$ to load $\mathrm{PdCl}_{4}{ }^{2-}$ by ion exchange, followed by rinsing with DI water to remove the physics' absorbed inks. The ELD of Cu was performed in a plating bath consisting of a 1:1 mixture of freshly prepared solution A and B. Solution A contains $\mathrm{NaOH}(12 \mathrm{~g} / \mathrm{L}), \mathrm{CuSO}_{4} \cdot 5 \mathrm{H}_{2} \mathrm{O}(13 \mathrm{~g} / \mathrm{L})$, and potassium sodium tartrate (29 $\mathrm{g} / \mathrm{L})$ in DI water. Solution B is a $\mathrm{HCHO}(9.5 \mathrm{~mol} / \mathrm{L})$ aqueous solution. After ELD, all samples were rinsed with DI water and dried by compressed air.

Characterization: The morphology of copper film was investigated by Scanning Electron Microscopy (SEM) (TM3000, Hitachi). The contact angle was measured by a water contact angle meter (C201, USA Kino Industry Ltd.). The bulk resistance was investigated by a 2point probe method with a Keithley 2400 Source meter. The sheet resistance was tested by a 4-point probe system (RTS-9, 4 PROBES TECH). The resistivity is calculated by using the measured sheet resistance and the thickness of metal films. 

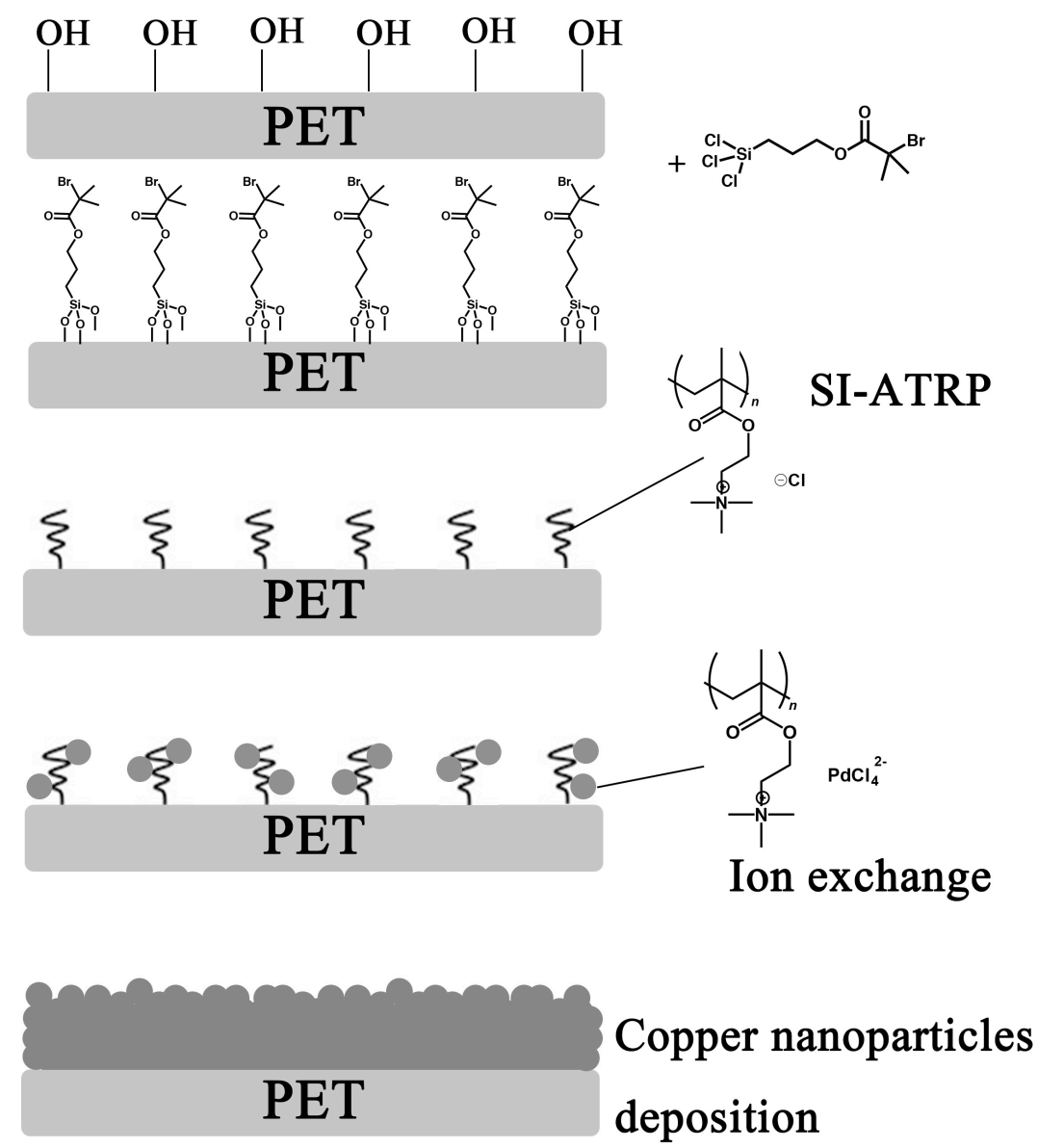

Figure 1. Schematic illustration of the process of preparing copper film on PET substrates.

The dimension is not drawn to scale.

\section{Results and discussion}

\subsection{Per-treated by Low Temperature Plasma}

The PET substrates were firstly treated in the low-temperature plasma for $5 \mathrm{~s}, 10 \mathrm{~s}, 20 \mathrm{~s}, 30 \mathrm{~s}$, $40 \mathrm{~s}, 50 \mathrm{~s}, 60 \mathrm{~s}$ and increasing to $120 \mathrm{~s}$, respectively. To investigate the density of active chemical groups, contact angles of the PET substrate were tested as shown in Figure 2. Integrally, the values of the contact angle decrease according to the treatment time increasing, and the treated surface changes to super-hydrophilic ultimately. The wettability of the surface appears to be increased strongly within the first 10 seconds of treatment. As the treatment time increasing to $60 \mathrm{~s}$, the fabrics change to super-hydrophilic and the water drops permeate 


\section{WILEY-VCH}

into the inner of fabrics, which attribute to the roughness and capillary effect caused by the particular structures of fabric. Any subsequent surface modification following longer treatment after $60 \mathrm{~s}$ was less important. The wettability of the surface can be confirmed that functional groups such as hydroxyl, carbonyl, carboxylic, amino and amines are formed on the surface of PET, which will effectively react with the trichlorosilane initiator molecules in the next step.

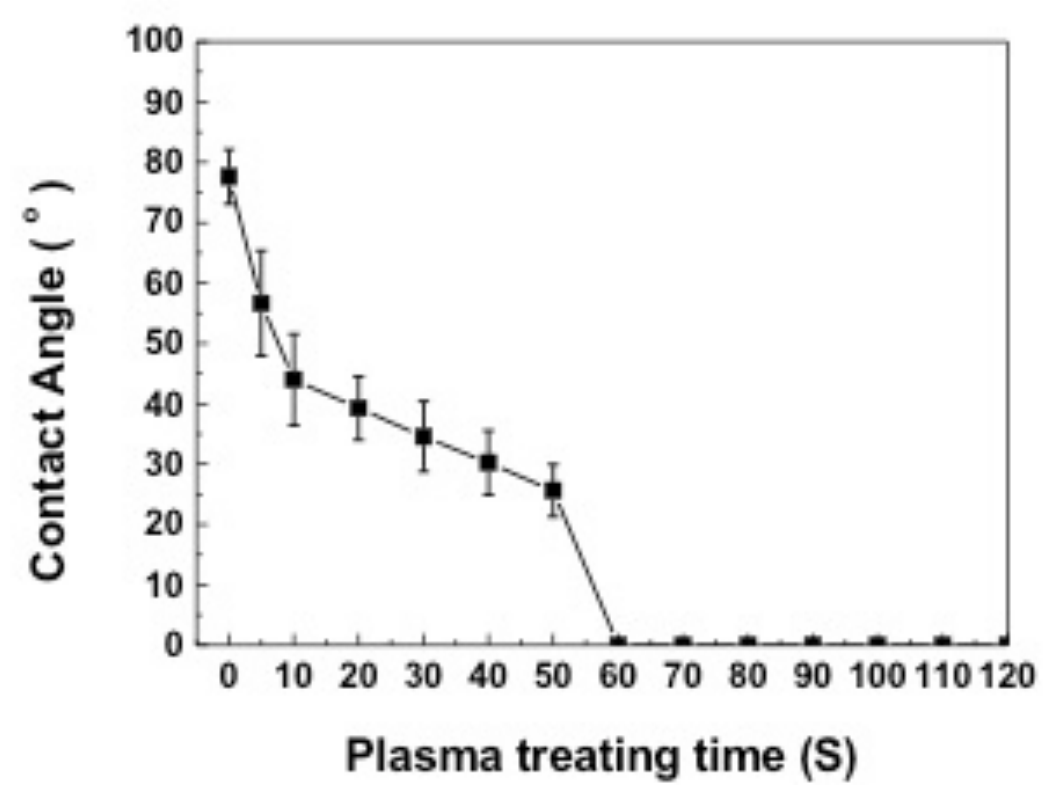

Figure 2. Water contact angles of PET fabrics treated by plasma with different time.

\subsection{Synthesis of Polymer Brushes}

As schematically illustrated in Figure 1, poly [2-(methacryloyloxy)ethyl-trimethylammonium chloride] (PMETAC) brushes were grafted from PET fabrics by surface-initiated ATRP. The main evidence of the successfully grafting process is confirmed by Fourier Transform Infrared Spectroscopy (FTIR). From Figure 3, PET fabrics without plasma treatment and grafted by PMETAC brushes were compared and some characterized peaks were analyzed and attributed. The new peak at $1634 \mathrm{~cm}^{-1}$ and the peak at $1171 \mathrm{~cm}^{-1}$ are attributed to the carbonyl groups and quaternary ammoniums groups $\left(\mathrm{QA}^{+}\right)$of PMETAC, respectively. 


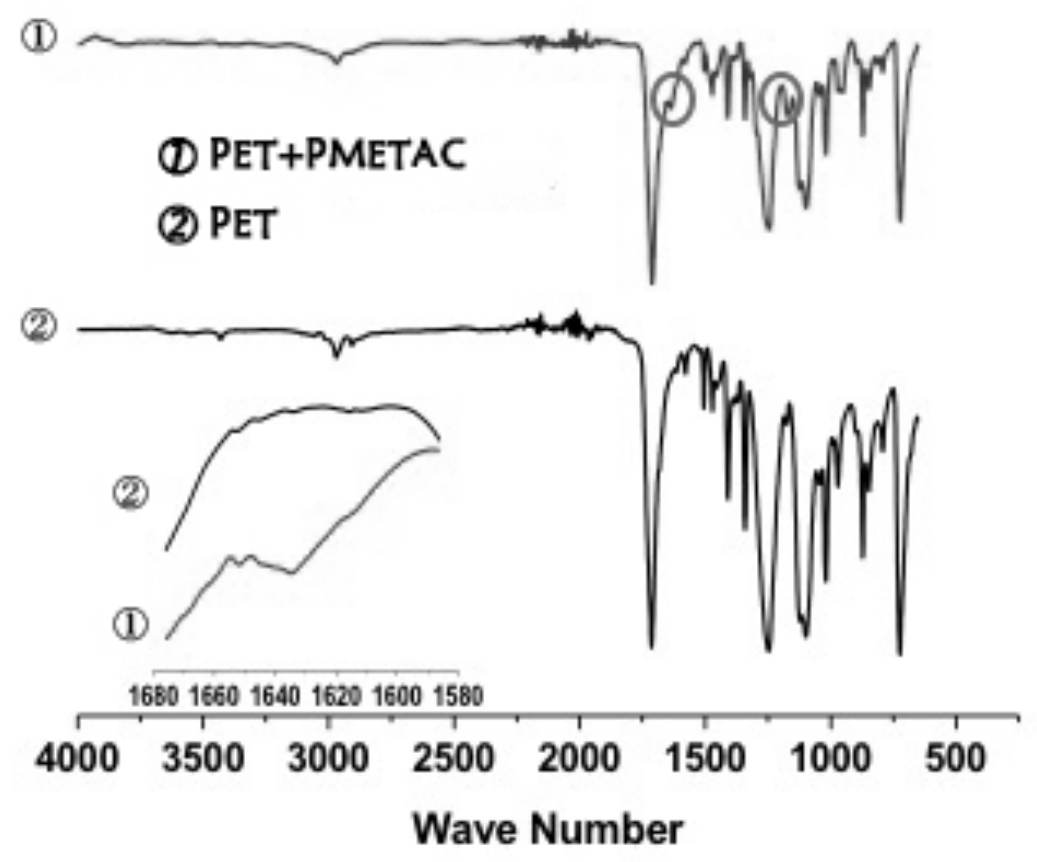

Figure 3. Reflectance FTIR spectra of PET fabrics modified by PEMATEC brushes (1) and control (2).

\subsection{Electroless Deposition}

The weights of fabric samples with the same size of before and after treatment were measured by an electronic weighing balance, respectively. The increasing percentage of deposit in weight was calculated by the following equation (1):

$$
\delta(\%)=\frac{W 2-W 1}{W 1} \times 100 \%
$$

Where $\delta$ is the weight increasing percentage $(\%), \mathrm{W}_{1}$ is the fabric original weight $(\mathrm{g})$, and $\mathrm{W}_{2}$ is the final plated fabric weight $(\mathrm{g})$. From Figure 4, it can be seen that longer plating results in heavier fabrics which are due to increase amount of plated metal. As typically electroless deposition, the copper ions are reduced in situ and the new resulting $\mathrm{Cu}^{0}$ nanocystal act as a seed layer for the subsequent electroless copper nanoparticles growth which, thus, the thickness of the coating has increased. 


\section{WILEY-VCH}



Figure 4. Weight increase of PET fabrics at different ELD plating times. The PET substrates are treated by plasma for $10 \mathrm{~s}, 30 \mathrm{~s}$, and $60 \mathrm{~s}$.
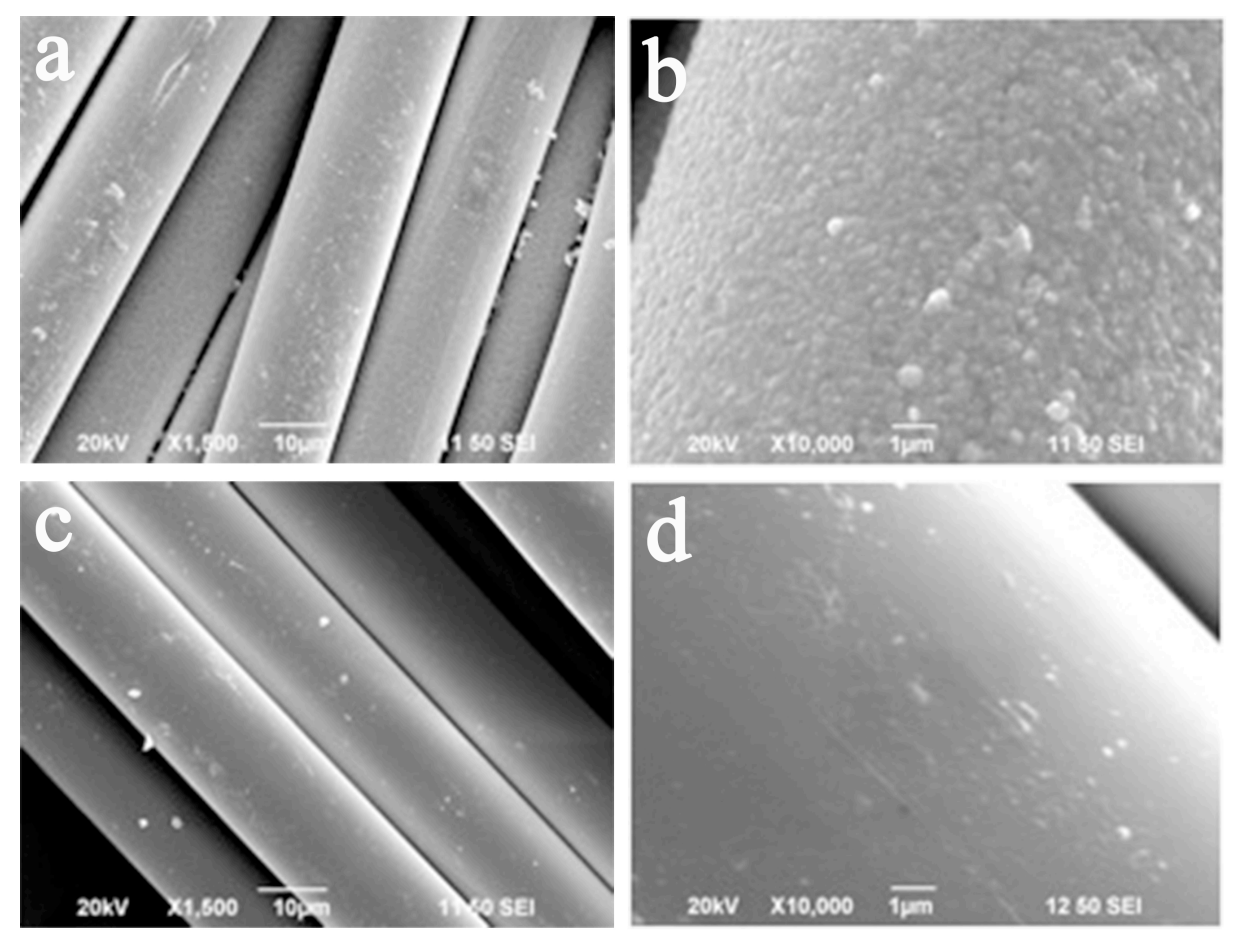

Figure 5. SEM photographs of copper coating on PET film with $(a, b)$ and without $(c, d)$ plasma treatment (Electroless plating time is 60 mins).

\subsection{Surface characterization}

The surface morphology of thin copper films may affect the electrical, mechanical, and 


\section{WILEY-VCH}

optical properties of PET fabrics. The morphology of the obtained copper coatings was characterized by scanning electron microscope (SEM) in detail. Figure4 shows the SEM photographs of the copper coating on PET fabrics with $(a, b)$ and pure PET fabrics $(c, d)$. In comparison with Figure 5a, there is a significant change on the surface of PET fibre after copper electroless deposition (Figure 5b).

After copper electroless depositing, it can be clearly observed that copper particles were uniformly and continuously dispersed on the surfaces of PET fibre. More importantly, it shows that the copper nanoparticles deposited not only on the upper surface of the fabrics, but also on the surface of inner fibres, and this integral and continuous coating will enhance electrical properties of the prepared metallic fabrics. From Figure $5 \mathrm{c}$ we can see that the copper nanoparticle film was compact and continuous on the polymer brushes modified fibres, which was pre-treated by plasma. But there are no observed particles depositing on the surface of the sample which was not treated by plasma, which indicated that the plasma Pretreatment is a significant decisive factor to the electroless deposition.

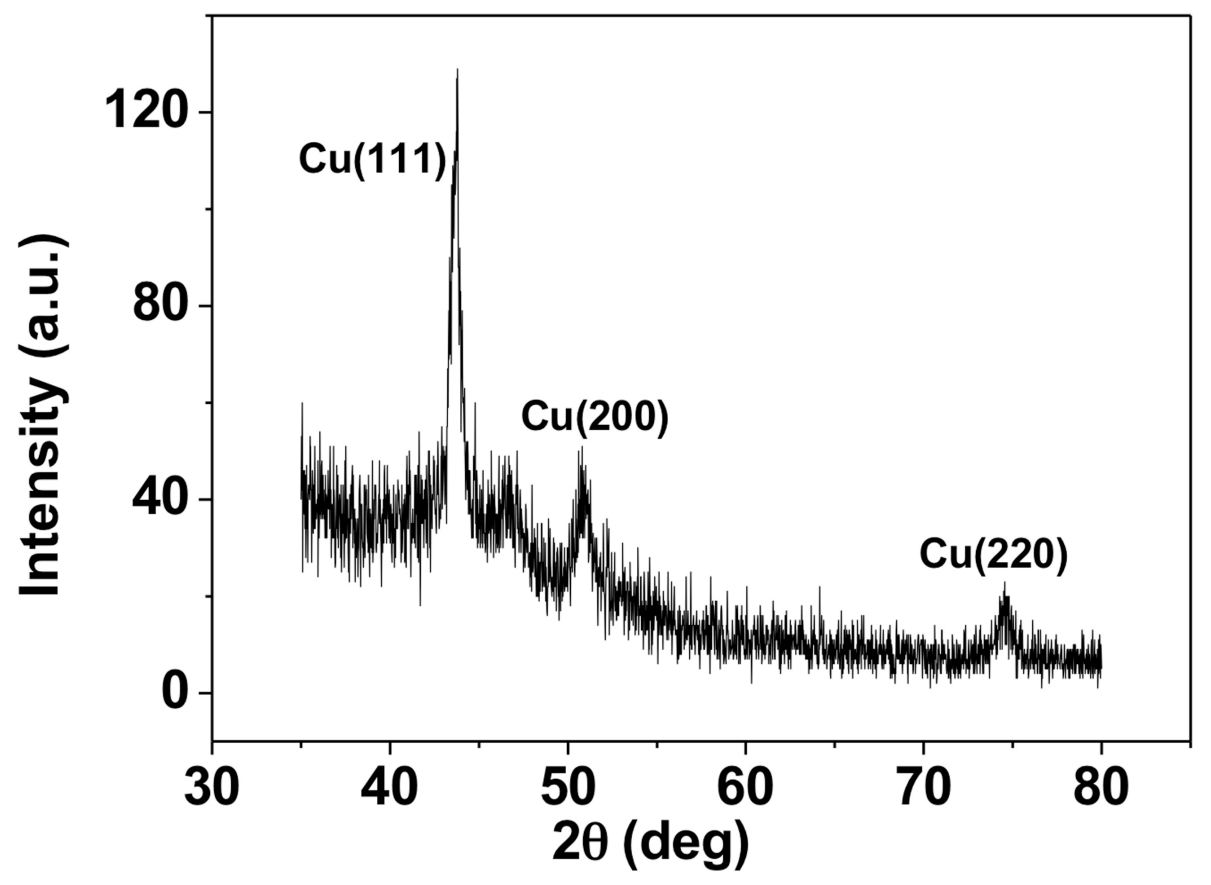

Figure 6. XRD analysis of Copper/PET composite.

XRD is a traditional and easy tool to determine the size and the shape of the unit cell for any 


\section{WILEY-VCH}

compound. Powder Diffraction Methods are useful for Qualitative analysis (Phase Identification), Quantitative analysis (Lattice parameter determination \& Phase fraction analysis) etc. Diffraction pattern gives information on translational symmetry-size and shape of the unit cell from Peak Positions and information on electron density inside the unit cell. It also gives information on deviations from a perfect particle, extended defects and micro strain from Peak Shapes \& Widths.

The X-ray diffraction patterns of copper particles on the polyester surface are shown in Figure 6. Three peaks at $2 \theta$ values of $43.6^{\circ}, 50.3^{\circ}$ and $74.2^{\circ}$ corresponded to the crystal faces of (111), (200) and (220) of planes of copper were observed and compared with the standard powder diffraction card of JCPDS. ${ }^{[13]}$ Based on The X-ray diffraction patterns, the particle average size of copper nanoparticles could be calculated from the broadening of the diffraction peak by the Scherrer formula:

$$
t=\frac{\mathrm{n} \lambda}{\mathrm{B} \cos \theta}
$$

$\mathrm{t}$ is the mean size of the ordered (crystalline) domains, which may be smaller or equal to the grain size; $\mathrm{n}$ is a dimensionless shape factor; $\lambda$ is the $\mathrm{X}$-ray wavelength; $\mathrm{B}$ is the line broadening at half the maximum intensity (FWHM), $\theta$ is the Bragg angle.

With respect to the $\mathrm{Cu}(111)$ main peak, the average size of copper particles was $31.06 \mathrm{~nm}$ according to the Scherrer equation. It can be identified that the deposited copper nanoparticles exhibited a characteristic face-centered cubic crystalline structure by the PDF card of the JADE-SCAN software, which could imply the perfect conductive property of copper-plated polyester fabrics. 


\section{WILEY-VCH}
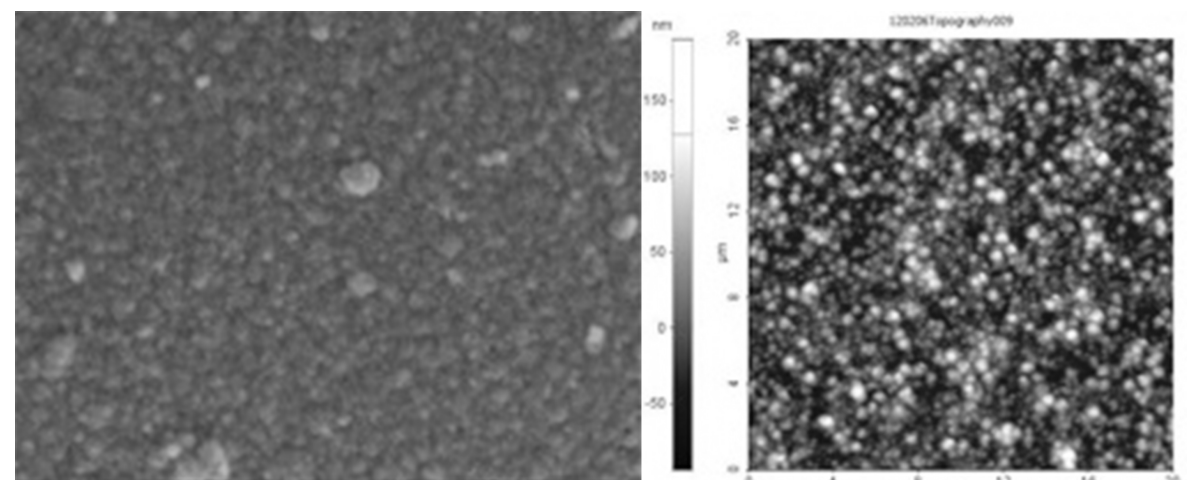

Figure 7. Topography images of $\mathrm{Cu}$ nanoparticles on PET substrate. Image on the left is observed by FE-SEM, and the right image is scanned by AFM.

As an intuitive measurement, the copper particles size is determined by FE-SEM (Figure 7 Left) and AFM (Figure 7 Right), respectively. It can be observed that the size of nanoparticles is even granular, which is around $30-50 \mathrm{~nm}$. The nanoparticles are compact and continuous on the PET surface with the compact arrangement, whose particular distribution should lead to high electronic conductive properties of this as-made composite. The AFM image (Figure 7 Right) indicates the surface morphology of deposited copper nanoparticles with plasma assistant technique. The $\mathrm{R}_{\mathrm{RMS}}$ (square roughness) of the $\mathrm{Cu}$-deposited surfaces (area: $20 \mu \mathrm{m} \times 20 \mu \mathrm{m}$ ) for the sample is estimated to be $28.6 \mathrm{~nm}$ which is a coincident with the FE-SEM image.

\subsection{Electric Conductivity and Washability}

It could be observed from Figure 8a that the electronic surface resistivity of PET fabrics decreases with increasing ELD time, reaching as low as $2 \Omega / \mathrm{sq}$ at $60 \mathrm{~min}$. That could be attributed to the quantity of copper nanoparticles increasing on the PET fabrics, which make the copper films more continuous. The most critical challenge of conductive fabrics is that the wash-ability and long-term durability under multiple cycles. For proof-of-concept purpose, we challenged the robustness by running through the washing cycles using simple hand washing and squeeze-drying. A sample of copper/PET conductive fabrics by physical deposition was also tested in the same washing cycles. It could be found that after 5 washing 


\section{WILEY-VCH}

and drying cycles, there is no obvious increase of surface resistivity on the sample fabricated by electroless deposition. This was basically attributed to the covalent bond between the surface and the grafted polymer brushes as well as to the nanometer-scale mechanical interlocking of the growing electroless plated layer within the brushes, which was provided by the polymer viscoelastic and high swelling intrinsic properties. A sample of $\mathrm{Cu} / \mathrm{PET}$ made by thermal evaporation was also tested by the same hand washing process, the surface resistivity was increasing dramatically resulting in flaking off during bending and rubbing, and cannot withstand washing tests. According to our results, plasma assistant polymer brushes were however, sufficient to bring good adhesion properties onto the substrates.
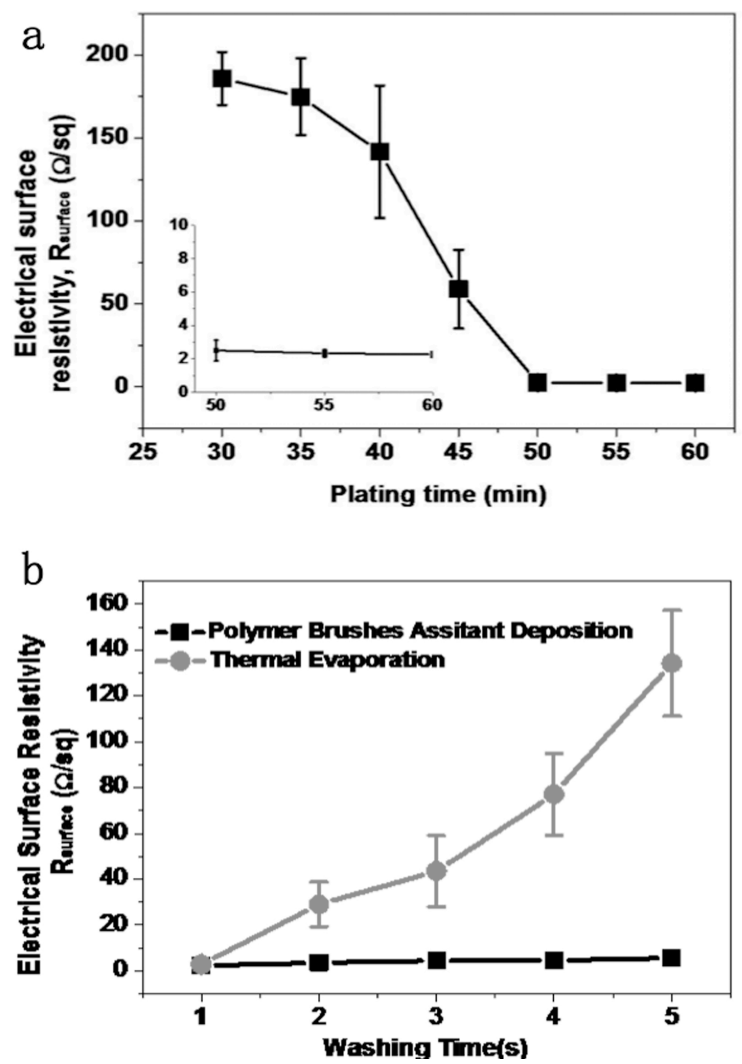

Figure 8. Conductivity tests of the prepared conductive PET fabrics with different plating time (a) and wash fitness test of the conductive PET fabrics (60 min plating) and samples of commercial cotton fabrics by thermal evaporation (b). The conductivity remains constant after 5 washing cycles by hand.

\subsection{Demonstration as electronic circuits}




\section{WILEY-VCH}

The most important application for conductive fabrics is used as an electric conductor in an electric circuit with the current system. To demonstrate this application in detail, a basic and simple circuit was constructed by bridging a $9 \mathrm{~V}$ battery and one electrical contact of a blue LED with manufactured conductive yarns and conductive fabrics (Figure 9b,c). When the contact between the LED and the battery was made, the LED turned on immediately and illuminated for more than 10 min until we disconnected the contact. The LED can be repeatedly switched ON and OFF by connecting and disconnecting the contact without loss of fidelity.



Figure 9. Digital images of electronic circuits. Initial PET yarn and as-made conductive yarn(a), Conductive yarn (b), and fabric (c) are used as electrical wire and substrate for powering a blue LED, respectively. The yarn or fabric was placed in contact with the battery LED without using extra conductive glue or paste.

\section{Conclusion}

In conclusion, we developed a novel process to prepare durable, flexible, and electrically conductive fibre \& fabrics. Firstly, the PET fabrics are pre-treated by plasma to generate active chemical groups, as conformed by significant decreases in contact angle and increase in surface energy. Then PMETAC brushes were grown from the active PET surface by surface initiated ATRP, which acts as a platform for ELD of copper particles depositing to form polymer-bridges copper/PET hierarchical structures subsequently. This in situ depositing approach ensures that the copper nanoparticles could distribute uniformly and continually on 


\section{WILEY-VCH}

the surface of the PET fabric structure. And these formatted conductive PET fabrics have a highly durable conductivity because the grafting polymer brushes act as effective bond layer leading to excellent electrical stability under bending, stretching, and washing. Such manufactured conductive fabrics can be used as an electrical conductor to powering a LED. In principle, this copper coating method could be extended to the coating of metals on other synthetic fabrics, polymer films, and bulk polymer materials. Such conductive fabrics could develop a wide variety of applications in wearable and flexible electronics, radiation and electromagnetic protection, energy, and biomedical industries, and it also provides an excellent alternative method to obtain a metal coating on polymer substrates ${ }^{[10,14]}$.

\section{Acknowledgements}

We would like to thank the support of the Hong Kong Polytechnic University, the EU Horizon 2020 and University of Manchester through projects with project codes 644268ETEXWELD-H2020-MSCA-RISE-2014, and AA01906. Also,we would like to thank revision and suggestion from Mr. Stephen Cowling.

Received: ((will be filled in by the editorial staff))

Revised: ((will be filled in by the editorial staff))

Published online: ((will be filled in by the editorial staff))

[1] A. Garcia, J. Polesel-Maris, P. Viel, S. Palacin, T. Berthelot, Advanced Functional Materials 2011, 21, 2096; M. S. Ersoy, E. Onder, Textile Research Journal 2014, 0040517514530025 ; E. G. Han, E. Kim, K. W. Oh, Synthetic Metals 2001, 123, 469; Y. Lu, S. Jiang, Y. Huang, Surface and Coatings Technology 2010, 204, 2829; Y. Lu, Q. Liang, W. Li, Materials Chemistry and Physics 2013, 140, 553; J.-S. Roh, Y.-S. Chi, T. J. Kang, S.-w. Nam, Textile Research Journal 2008, 78, 825; Y. Wang, T. Hua, B. Zhu, Q. Li, W. Yi, X. Tao, Smart materials and structures 2011, 20, 065015 . 


\section{WILEY-VCH}

[2] R. Guo, S. Jiang, C. Yuen, M. Ng, Journal of The Textile Institute 2012, 103, 1267.

[3] E. Kim, E. Han, K. Oh, J. Na, Journal of Applied Physics 2000, 87, 4984.

[4] K. Rajendrakumar, G. Thilagavathi, Indian Journal of Fibre \& Textile Research 2012, $37,133$.

[5] X. Wang, C. Yan, H. Hu, X. Zhou, R. Guo, X. Liu, Z. Xie, Z. Huang, Z. Zheng, Chemistry-An Asian Journal 2014, 8, 2170.

[6] X. Wang, H. Hu, Y. Shen, X. Zhou, Z. Zheng, Advanced Materials 2011, 23, 3090.

[7] Y. Yu, J. Zeng, C. Chen, Z. Xie, R. Guo, Z. Liu, X. Zhou, Y. Yang, Z. Zheng, Advanced Materials 2014, 26, 810.

[8] A. P. Chen, P. W. Hwang, C. W. Lin, T. A. Lin, Y. Y. Chuang, J. H. Lin, Applied Mechanics and Materials 2013, 365, 1173; Q. Liu, Z. Zhou, M. Xia, Y. Tao, K. Liu, D. Wang, RSC Advances 2014, 4, 40788.

[9] R. Guo, S. Jiang, C. Yuen, M. Ng, J. Lan, Fibres and Polymers 2013, 14, 459.

[10] R. Guo, Y. Yu, Z. Xie, X. Liu, X. Zhou, Y. Gao, Z. Liu, F. Zhou, Y. Yang, Z. Zheng, Advanced Materials 2013, 25, 3343.

[11] K. D. Pangilinan, A. C. C. de Leon, J. D. Mangadlao, E. Baer, R. C. Advincula, Macromolecular Materials and Engineering 2016, on line.

[12] N. Rajender, K. I. Suresh, Macromolecular Materials and Engineering 2016, 301, 81.

[13] X. Liu, Y. Li, Z. Zheng, F. Zhou, RSC Advances 2013, 3, 341.

[14] X. Liu, X. Zhou, Y. Li, Z. Zheng, Chemistry - An Asian Journal 2012, 7, 862. 


\section{WILEY-VCH}

Active poly [2-(methacryloyloxy)ethyl-trimethylammonium chloride] (PMETAC) brushes is grafted from the PET surface by surface initiated atom transfer radical polymerization, as a platform for copper nanoparticles deposited, which can increase the adhesion between conductive coating and fibres.



